\title{
Pyrin, Product of the MEFV Locus, Interacts With the Proapoptotic Protein, Siva
}

\author{
BANU BALCI-PEYNIRCIOGLU,' ANDREA L. WAITE, ${ }^{2}$ CHUNBO HU, ${ }^{2}$ NEIL RICHARDS, ${ }^{2}$ \\ ANN STAUBACH-GROSSE, ${ }^{2}$ ENGIN YILMAZ,' AND DEBORAH L. GUMUCIO ${ }^{2 *}$ \\ 'Department of Medical Biology, Faculty of Medicine, Hacettepe University, Ankara, Turkey \\ ${ }^{2}$ Department of Cell and Developmental Biology, University of Michigan Medical School, Ann Arbor, Michigan
}

\begin{abstract}
Mutations in pyrin cause the autoinflammatory disorder familial Mediterranean fever (FMF), a syndrome characterized by sporadic and unpredictable attacks of fever and localized severe pain. Currently, it is not clear how attacks are triggered, nor why they spontaneously resolve after 2 or 3 days. In fact, the cellular function of the pyrin protein and the molecular underpinnings of its malfunction in FMF have so far eluded clear definition. The identification of pyrin-interacting proteins has the potential to increase our understanding of the cellular networks in which pyrin functions. Previous reports have established that pyrin interacts with the apoptotic protein ASC, the cytoskeletal adaptor protein PSTPIPI, the inflammatory caspase, Caspase-I and certain forms of the cytosolic anchoring protein I4-3-3. Here, we report that pyrin also interacts with Siva, a pro-apoptotic protein first identified for its interaction with the cytosolic tail of CD27, a TNF family receptor. The interaction between pyrin and Siva involves the C-terminal B30.2/rfp/SRPY domain of pyrin and exon I of Siva. We show that Siva and pyrin are indeed co-expressed in human neutrophils, monocytes, and synovial cells. Furthermore, using a novel protein/ protein interaction assay, we demonstrate that pyrin can recruit Siva to ASC specks, establishing a potential platform for intersection of ASC and Siva function. Finally, we show that pyrin modulates the apoptotic response to oxidative stress mediated by Siva. Thus, the Siva-pyrin interaction may be a potential target for future therapeutic strategies.

J. Cell. Physiol. 216: 595-602, 2008. (C) 2008 Wiley-Liss, Inc.
\end{abstract}

Familial Mediterranean fever (FMF) is classified as an autosomal recessive disease that is characterized by sporadic attacks of fever and localized inflammation (Livhen et al., 1997). Although FMF is prevalent among eastern Mediterranean populations, mainly non-Ashkenazi Jews, Armenians, Turks and Arabs, it is observed throughout the world due to extensive population movements of the 20th century (Schaner and Gumucio, 2005). The FMF gene, which is located on the short arm of chromosome 16 and symbolized as 'MEFV' (for mediterranean fever), encodes a 78I amino acid protein named pyrin or märenostrin (International FMF Consortium; French FMF Consortium) herein referred to as pyrin. Pyrin is expressed primarily in neutrophils, eosinophils, cytokine-activated monocytes and some fibroblasts (Centola et al., 1998; Diaz et al., 2004). Based on the nature of FMF attacks, it has been speculated that wild-type pyrin acts as a regulator of the inflammatory response, though the precise nature of this regulatory activity remains to be identified.

Previous identification of several pyrin-interacting proteins, including ASC (Richards et al., 200I) and PSTPIPI (Shoham et al., 2003), has led to the conclusion that pyrin participates in at least three cellular pathways that are connected to inflammation: cell death, cytokine secretion and cytoskeletal signaling. ASC acts as an adaptor protein to promote the assembly of a variety of large multi-protein complexes that mediate IL-I $\beta$ processing (Martinon et al., 2002). ASC also provides a central platform for a cell death pathway that involves the generation of a large cytosolic perinuclear aggregate called a "speck" (Masumoto et al., 1999) or pyroptosome (Fernandes-Alnemri et al., 2007). A second pyrin-interacting protein, PSTPIPI, is also thought to act as an adaptor. PSTPIPI links PEST phosphatases to their substrates, cytoskeletal signaling proteins such as WASP, c-Abl, and Crk (Cong et al., 2000). Importantly, mutations in PSTPIPI cause PAPA syndrome (pyogenic arthritis, pyoderma gangrenosum and acne) (Wise et al., 2002), which like FMF, is an autoinflammatory syndrome involving severe reactive skin and joint inflammation. The PAPA mutations reduce PSTPIPI binding to PEST phosphatases and increase PSTPIPI binding to pyrin, suggesting that pyrin and PSTPIPI are members of a common pathway that controls inflammatory reactions (Shoham et al., 2003). Recently, additional reports have emerged indicating that pyrin also interacts directly with Caspase-I (Chae et al., 2006), the major activator of IL-I $\beta$, and with certain isoforms of I4-3-3 (Jeru et al., 2005), an interaction that has the potential to control the compartmentalization of pyrin in nucleus or cytoplasm.

Here, we report the identification of a fifth pyrin-interacting protein: the pro-apoptotic protein, Siva. Siva was previously characterized as a protein that interacts with the cytoplasmic tail of several TNF receptor family members (CD27, GITR, OX40, and 4-IBB) (Prasad et al., I997; Spinicelli et al., 2002). Siva also interacts with and neutralizes some of the anti-apopototic BCL-2 family members (Xue et al., 2002; Chu et al., 2004). Siva has been implicated in cell death following Coxsackie virus infection (Henke et al., 200I) and has been identified as a p53 target gene (Fortin et al., 2004) that can enhance apoptosis in response to cisplatin and other cellular

Banu Balci Peynircioglu and Andrea L. Waite contributed equally to this study.

Contract grant sponsor: $\mathrm{NIH}$;

Contract grant number: Al53262.

*Correspondence to: Deborah L. Gumucio, PhD, Department of Cell and Developmental Biology, University of Michigan Medical School, 109 Zina Pitcher Place, 2045 BSRB, Ann Arbor, MI 481092200. E-mail: dgumucio@umich.edu

Received 20 September 2007; Accepted 4 February 2008

DOI: 10.1002/jcp.21435 
toxins (Chu et al., 2005). The establishment of a direct physical connection between Siva and pyrin further broadens the realm of pyrin's potential influence on cellular inflammatory and apoptotic signaling.

\section{Materials and Methods}

Yeast two-hybrid screen

Screening for pyrin-interacting proteins was performed as previously described (Richards et al., 200I). Full-length human pyrin was used as the bait to screen a cDNA library $\left(2 \times 10^{6}\right.$ clones $)$ prepared from human neutrophil RNA isolated from a gouty knee. A total of I 32 clones were isolated and confirmed as bone fide pyrin interacting proteins using the protocol previously described (Richards et al., 200I). One of these encoded the entire coding region and 5'UTR of Siva-I.

\section{Cell culture and transfection}

COS-7 and human embryonic kidney 293T cells were grown in DMEM supplemented with $10 \%(\mathrm{v} / \mathrm{v})$ FBS. Cells were transiently transfected by using FuGENE 6 (Roche Applied Science, Indianapolis, IN). COS-7 were used for immunofluorescence staining and 293T cells were used for immunoprecipitation.

For monocyte/neutrophil isolation, heparinized blood from healthy adult volunteers was mixed with $6 \%$ dextran in PBS at a ratio of $5: I$ and was allowed to settle at $37^{\circ} \mathrm{C}$ for $30 \mathrm{~min}$. The buffy coat was layered onto the Ficoll-Hypaque (Pharmacia) density gradient and was centrifuged for $15 \mathrm{~min}$ at $1,500 \mathrm{rpm}$ at room temperature. Neutrophils and monocytes were recovered from the pellet of the gradient by hypotonic lysis of contaminating erythrocytes using $\mathrm{H}_{2} \mathrm{O}$. Neutrophils were greater than $98 \%$ pure, as assessed by May Giemsa staining. Mononuclear cells $\left(2 \times 10^{6}\right)$ were seeded in six-well plates in $2 \mathrm{ml}$ of serum-free RPMI medium I,640 for I h. Nonadherent cells were removed by aspiration and wells were washed with PBS.

Human synovial cells (fifth to eighth passage) were kindly provided by Dr. David Fox (Department of Rheumatology, University of Michigan). Synoviocytes were stimulated by incubation with LPS $(100 \mathrm{U} / \mathrm{ml})$.

\section{Plasmids}

Full-length Siva-I, image clone Siva-2 (open biosystems-clone ID 3860364) and deletion constructs were subcloned into the pEGFP-C2 (Clontech, Mountain View, CA) vector. All myc-tagged pyrin constructs were cloned into the PCMVTag3a (Stratagene, La Jolla, CA) vector.

\section{Expression analysis}

Semiquantitative PCR analysis was performed on total RNA isolated from neutrophils, synovial cells, monocytes, SW-I3, SW-480, THP-I, J774, and COS-7 cells. Primer sequences used for PCR were; F: 5'CTCCTCCACCAGAAGTCAG 3',

R 5'AGCTCTGGAACATTGAACATT $3^{\prime}$ for pyrin,

F: 5'GCCTCCTTTGACTCCTTGA AGC 3', R: 5'

TTCTGCCTCTCACGAAACTCCTC $3^{\prime}$ for PSTPIPI, F: $5^{\prime}$

CTTCTACCTGGAGACCTACGGC 3', R: $5^{\prime}$

TCTTGCTTGGGTTGGTGGG $3^{\prime}$ for ASC and F: $5^{\prime}$

CGAGAAGACCAAGCGACTCC 3', R: 5'

TGAGGAACAGGCAATGGACG $3^{\prime}$ for Siva- I, F: $5^{\prime}$

CGAGAAGACCAAGCGACTCC 3', R: 5'

TGAGGAACAGGCAAT GGACG $3^{\prime}$ for Siva-2. Primer sequences used for control HPRT PCR were; F: $5^{\prime}$

TGGCGTCGTGATTAGTGATG $3^{\prime}$ and R: $5^{\prime}$

AATCCAGCAGGTCAGCAAAG 3'.

\section{Antibodies}

Rabbit anti-myc polyclonal antibody was obtained from Sigma, St. Louis, MO, and mouse anti-GFP monoclonal antibody was obtained from Clontech. Fluorescent secondary antibody AF 568 goat anti-rabbit was purchased from Molecular Probes. Horseradish peroxidase-conjegated antibodies used for Western blot (goat anti-mouse and mouse anti-myc) were purchased from Santa Cruz Biotechnology, Santa Cruz, CA.

\section{Immunoprecipitation and Western blot analysis}

293T cells were transiently transfected with different pyrin, Siva- I and Siva- 2 constructs. After $24 \mathrm{~h}$, cells were lysed in $10 \mathrm{mM}$ Tris- $\mathrm{HCl}$ pH 7.4, $300 \mathrm{mM} \mathrm{NaCl}, 2$ mM EDTA, 0.5\% Triton X-100 lysis buffer in the presence of Complete Mini EDTA-Free Protease Inhibitor Cocktail (Roche). Cells were incubated with anti-myc antibody which was crosslinked to protein A-G sepharose beads for $16 \mathrm{~h}$ at $4^{\circ} \mathrm{C}$. After washing the beads, bound proteins were subjected to SDS/PAGE and transferred to nitrocellulose membranes for detection with appropriate antibodies.

\section{Immunofluorescence staining}

After transfection, cells were fixed in $4 \%$ paraformaldehyde in PBS, permeabilized by using $0.2 \%$ Triton X-100 in PBS and blocked in $10 \%$ goat serum, I\% BSA in PBS for I h. After antibody treatment, DAPI staining was performed and cells were visualized with a Nikon E800 immunofluorescence microscope.

\section{ASC speck assay}

COS-7 cells were co-transfected with ASC, pyrin and Siva constructs. After transfection $(24 \mathrm{~h})$ immunofluorescence and DAPI staining (to stain nuclei) were carried out. Co-localization of Siva constructs with pyrin in ASC specks was then observed.

\section{Apoptosis assay}

The hydrogen peroxide apoptosis sensitization assay was carried out using a slight modification of a protocol previously used to detect Siva-mediated apoptosis (Cao et al., 200I). COS-7 cells were plated in 24-well plates and transfected with Siva-I, Siva-2, and/or various forms of wild-type or mutant pyrin. Forty hours after transfection, cells were treated with I $\mathrm{mM} \mathrm{H}_{2} \mathrm{O}_{2}$ for $2 \mathrm{~h}$, then washed with culture medium (DMEM $+10 \%$ FBS) and cultured for an additional $20 \mathrm{~h}$. Adherent and non-adherent cells were collected and stained with trypan blue. Live and dead cells were counted using a hemacytometer, and the percentage of apoptotic cells was calculated. The transfections were repeated four times and all counting was done by an investigator blinded as to the identity of the samples.

\section{Statistical analysis}

Statistical analysis was carried out using GraphPad Prism software. $P$ values were calculated using an unpaired two-tailed $t$ test, and values of $<0.05$ were considered to be significant.

\section{Results}

\section{Identification of Siva as pyrin binding protein}

We previously reported the generation of a human neutrophil cDNA library which we employed in a yeast two hybrid screen to identify ASC as a pyrin-interacting protein (Richards et al., $200 \mathrm{I}$ ). From the same screen, we isolated a single clone which, upon sequence analysis, proved to represent full length Siva-I. The human Siva gene is localized to chromosome 14q32-33 and is organized into four exons that code for the full-length Siva-I transcript. An alternate splice form, Siva-2, has also been described that lacks the exon 2 coding sequence (Py et al., 2004). The full-length form of Siva, Siva- $I$, is a $I 75$ amino acid protein that contains a conserved amphipathic helical region at its $\mathrm{N}$-terminus (partially missing in Siva-2) and a cysteine-rich $\mathrm{C}$-terminus that binds zinc and may adopt a zinc finger-like conformation (Nestler et al., 2006). 
Expression of Siva, pyrin, and other pyrin-interacting proteins

Siva was first isolated from T-cells as a CD27 binding protein, but expression of Siva is more widespread (Jacobs et al., 2007). Since the expression of pyrin is restricted to myeloid cells and certain fibroblast cell types (Centola et al., 1998; Diaz et al., 2004), we examined these particular cells as well as several additional cell lines for co-expression of pyrin and Siva message. The expression of mRNA encoding two other pyrin-interacting proteins, ASC and PSTPIPI, were also queried. We confirmed that neutrophils, monocytes, and synoviocytes, as well as SW-I3, SW-480, and THP-I cells all express the relevant messages, supporting the potential for physical interaction of these proteins (Fig. I). In addition, we found that none of these transcripts can be found in 293T cells (data not shown), indicating that these cells are an appropriate line to use to investigate the binding of pyrin and Siva independently of other known interacting proteins.

\section{Siva binds pyrin by immunoprecipitation}

Once it was established that pyrin and Siva are expressed in several relevant cell types, we set out to confirm the interaction between Siva and pyrin by immunoprecipitation. Myc-tagged pyrin and GFP-tagged Siva-I or Siva-2 were co-transfected into 293T cells. Immunoprecipitation was accomplished with anti-myc and blots were probed with anti-GFP. Both Siva-I and Siva-2 proteins were co-precipitated by antibodies to epitope-tagged pyrin (Fig. 2, lanes 3 and 4 respectively) but not by the negative control protein lacZ-myc (Fig. 2, lanes 2 and 5 respectively). Although expression of Siva-I and Siva-2 in

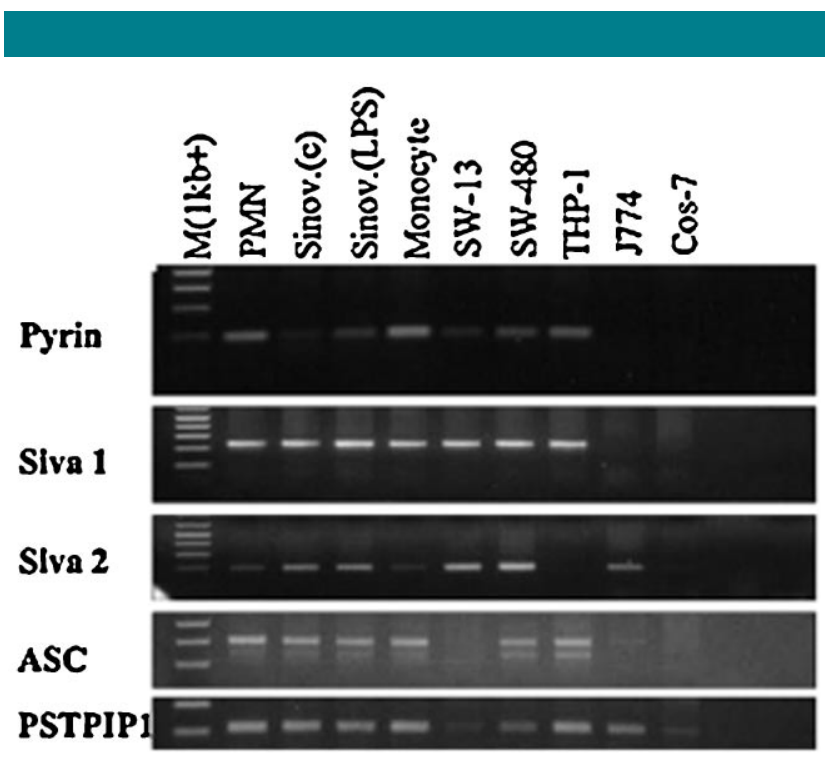

\section{HPRT}

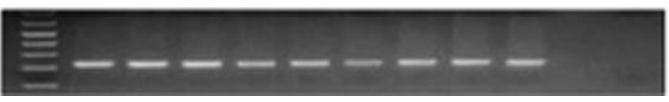

Fig. I. Endogenous expression of pyrin and pyrin-binding proteins. Native expression of pyrin, Siva-I, Siva-2, ASC, PSTPIPI and the housekeeping gene HPRT was examined in a variety of cell types. RT-PCR analysis was performed on total RNA from freshly isolated PMNs, synoviocytes (both unstimulated and stimulated with LPS), and monocytes, as well as SW-I3, SW-480, THP-I, J774, and COS-7 cells. Pyrin and Siva-I were detected in all cells with the exception of J774 and COS-7. Siva-2 expression was found in all cell lines except THP-I and COS-7. ASC was expressed in all cell lines except SW-I3, J774, and COS-7, while PSTPIPI was expressed in all cell lines.

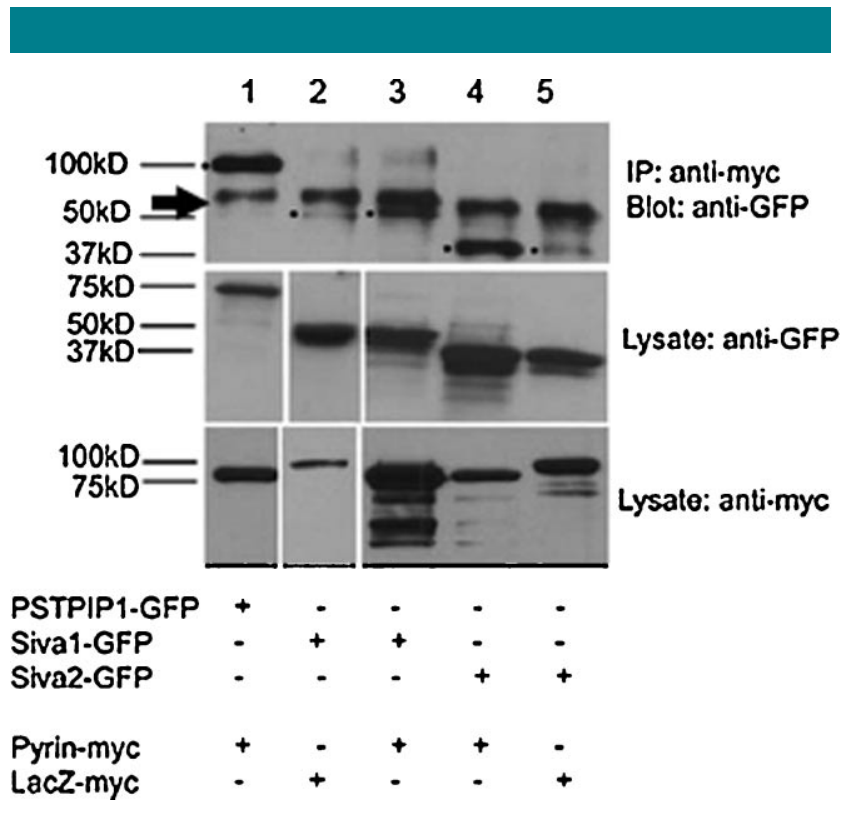

Fig. 2. Pyrin binds to Siva-I and Siva-2. 293T cells were co-transfected with GFP-tagged versions of Siva-I and Siva-2 and myc-tagged pyrin. Cell lysates were tested for expression of transfected proteins, and then immunoprecipitated using $\alpha$-myc polyclonal antibody (Sigma). Proteins were detected by Western blot using $\alpha$-GFP monoclonal antibody (Clontech). Small black dots to the left of the lanes indicate the expected location of co-precipitated proteins. Co-precipitation of pyrin and PSTPIP I was reconfirmed as a control (lane I). Full length pyrin-myc precipitated both Siva-I-GFP (lane 3) and Siva-2-GFP (lane 4) but not the control protein lacZ-myc (lanes 2 and 5 respectively). The band seen in all lanes of the top part (arrow) is a background band due to the $\alpha$-myc antibody used for immunoprecipitation.

lysates was similar (Fig. 2, center part, lanes 2 and 3 for Siva-I, lanes 4 and 5 for Siva-2), Siva-2 was reproducibly precipitated more efficiently than Siva-I, suggesting a stronger binding interaction between pyrin and Siva- 2 .

\section{Binding domains of pyrin and Siva}

To identify the domains in pyrin that interact with Siva, immunoprecipitation was performed using a set of deletion constructs of myc-tagged pyrin and GFP tagged Siva cotransfected into 293T cells (Fig. 3). Interestingly, the size of Siva varied slightly from lane to lane in the gel (Fig. 3A-C, center parts), possibly indicating post-translational modification of the protein; indeed, Siva is known to be phosphorylated by Arg kinase (Cao et al., 200I), and this may be highly context-dependent. Siva-I and Siva-2 were both immunoprecipitated using pyrin constructs lacking exon I, which encodes the pyrin domain or PyD (Fig. 3A,B, lanes 2 and $3)$. However, when IP was performed using pyrin constructs that lack other regions of the protein, a marked reduction in binding efficiency was seen. Of particular interest is the fact that the lowest binding levels were observed with pyrin exons I-9, which is missing the C-terminal B30.2 domain (also called the SPRY or rfp domain) encoded by exon 10 (Fig. 3A,B, lane I). These data indicate that the B30.2 domain plays an important role in the interaction of pyrin with Siva-I and Siva-2, although binding may be stabilized by other regions of the pyrin molecule.

Conversely, to determine the binding domain of Siva responsible for its interaction with pyrin, IP was performed using deletion constructs of GFP-tagged Siva and myc-tagged full length pyrin in co-transfected 293T cells. GFP-Siva exon I 
A

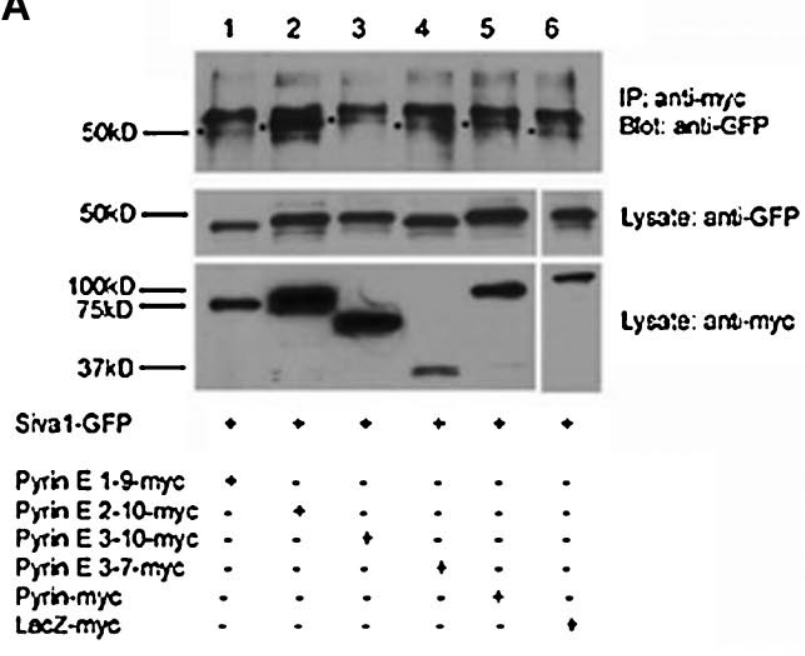

B

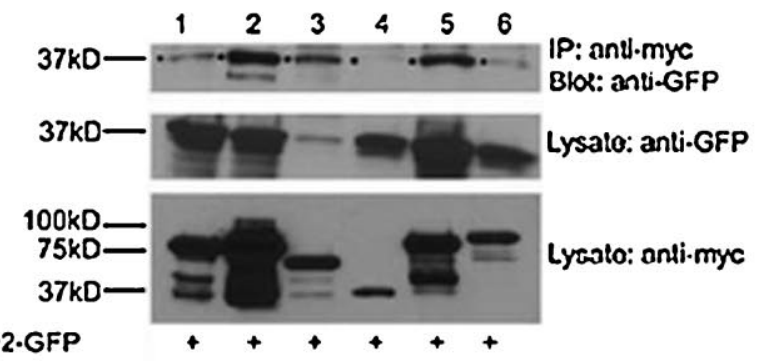

Siva2-GFP

Pyrin E 1.9.my'c Pyrin E 2-10-myc

Pyrin E 3-10-myc

Pyrin E 3.7-my'c

Pyrin-myc

LacZ-myc

C

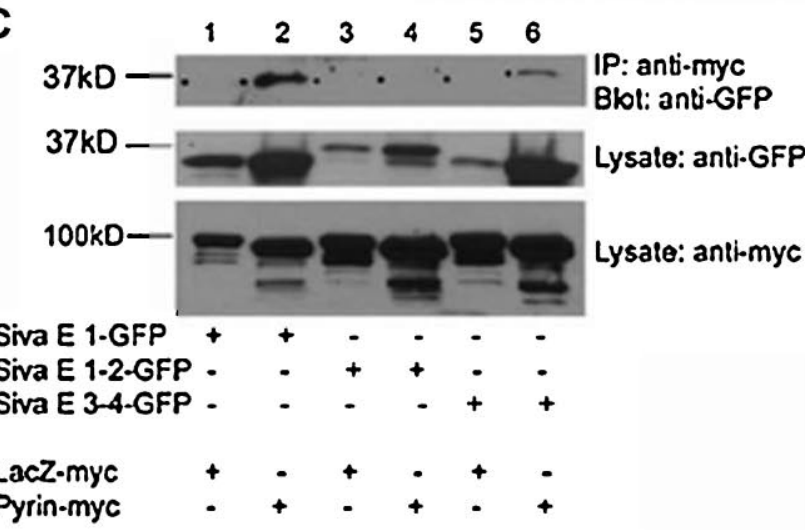

Fig. 3. Regions of pyrin involved in binding to Siva-I and Siva-2. A: Siva-I was immunoprecipitated with several different portions of pyrin. Strong binding was observed with pyrin exons 2-10-myc (lane 2), but binding to pyrin exons I-9 was very weak (lane I). B: Siva-2 precipitated strongly with myc-tagged pyrin exons 2-10 (lane 2) and 3-10 (lane 3), but not with exons 3-7 (lane 4) and very weakly with pyrin exons I-9 (lane I). C: Myc-tagged pyrin was co-transfected with GFP-tagged deletion constructs of Siva. Pyrin precipitated Siva exon I (lane 2), but binding was inhibited by the addition of Siva exon 2 (lane 4). Siva exons 3-4 were also weakly precipitated by pyrin (lane 6). Dots to the left of the lanes indicate expected sizes of precipitated proteins. was co-precipitated with pyrin (lane 2), while a construct encoding GFP-Siva exons 3-4 was much less efficiently co-precipitated (Fig. 3C, lane 6). Interestingly, precipitation of the Siva-I construct that included both exons I and 2 (Fig. 3C, lane 4) was less efficient than was Siva exon I alone (Fig. 3C, lane 2), suggesting a potential inhibitory effect of Siva's exon 2 on this interaction. This may explain why Siva-2, which lacks exon 2, appears to bind pyrin with higher affinity than Siva-I (Fig. 2). These data together indicate that the exon I coding domain of Siva is the primary region involved in the interaction of Siva proteins with pyrin.

\section{Co-localization of pyrin and Siva in cells}

To further confirm and explore the interaction between Siva and pyrin, we used immunofluorescence in co-transfected cells. Transfection of COS-7 cells with full-length pyrin linked to different epitope tags (myc-pyrin and GFP-pyrin) revealed a cytosolic distribution of the transfected pyrin protein

(Figure 4A, pyrin-myc shown), as previously demonstrated (Richards et al., 200I; Diaz et al., 2004). However, GFP-tagged Siva-I was predominantly nuclear, frequently localizing to discrete aggregates within the nucleus (Fig. 4B,C). GFP-tagged Siva- 2 was again observed in the nuclear compartment, but was also detectable in the cytoplasm, often in stress fiber-like structures (Fig. 4D,E). To test whether Siva constructs might localize predominantly to the cytosol in the early stages after transfection, when protein concentrations are lower, we transfected cells with GFP-tagged Siva-I and Siva-2 constructs and examined the cells at 6,9 , and $12 \mathrm{~h}$ after transfection. In all cases, Siva-I was found to be nuclear, while Siva- 2 was both cytoplasmic and nuclear (data not shown). Thus, at least in transfected cells, Siva prefers the nuclear environment.

When cells were co-transfected with pyrin and Siva-I, the two proteins appeared to maintain their distinct

compartmentalization (nuclear Siva-I and cytosolic pyrin; data not shown). When pyrin and Siva-2 were co-transfected, the two proteins showed a clear cytosolic co-localization on the stress fiber like structures and on the cellular membrane (Fig. 4F-K). These studies confirm that pyrin and Siva-2 are expressed in the same cellular compartment, which makes binding possible. However, this experimental technique failed to confirm expression of Siva-I and pyrin in the same cellular compartment.

Though our co-transfection experiments in COS-7 cells suggested that Siva-I is exclusively nuclear, Siva-I has been detected outside of the nucleus in other studies (Xue et al., 2002; Chu et al., 2005; Jacobs et al., 2007). We suspected therefore, that the trafficking of Siva-I may be highly regulated and that this protein may be capable of rapidly entering and leaving the cytosol in particular contexts. Therefore, we sought a method to detect small amounts of Siva-I that might exit the nucleus and test whether, once in the cytosol, a Siva-I/pyrin interaction could be demonstrated. For this purpose, we took advantage of another pyrin-interacting protein, ASC. ASC and pyrin interact via an $\mathrm{N}$ terminal PyD present in both molecules (Richards et al., 200I). Importantly, when cells are transfected with ASC, characteristic cytosolic speck-like aggregates are formed within $24 \mathrm{~h}$ (Masumoto et al., 1999; Richards et al., 200 I). If pyrin is co-transfected with ASC, pyrin is also drawn to the speck compartment. This requires exon I of pyrin, a region of the pyrin molecule that appears to be dispensable for its interaction with Siva. We therefore examined whether speckassociated pyrin could trap cytosolic Siva-I in cells triply transfected with myc-pyrin, untagged ASC, and EGFP-tagged Siva-I. Indeed, in triply transfected cells, both Siva-I and Siva-2 proteins could be observed in ASC specks by immunofluorescence microscopy (Fig. 5). In the absence of pyrin, neither Siva isoform was associated with specks, 

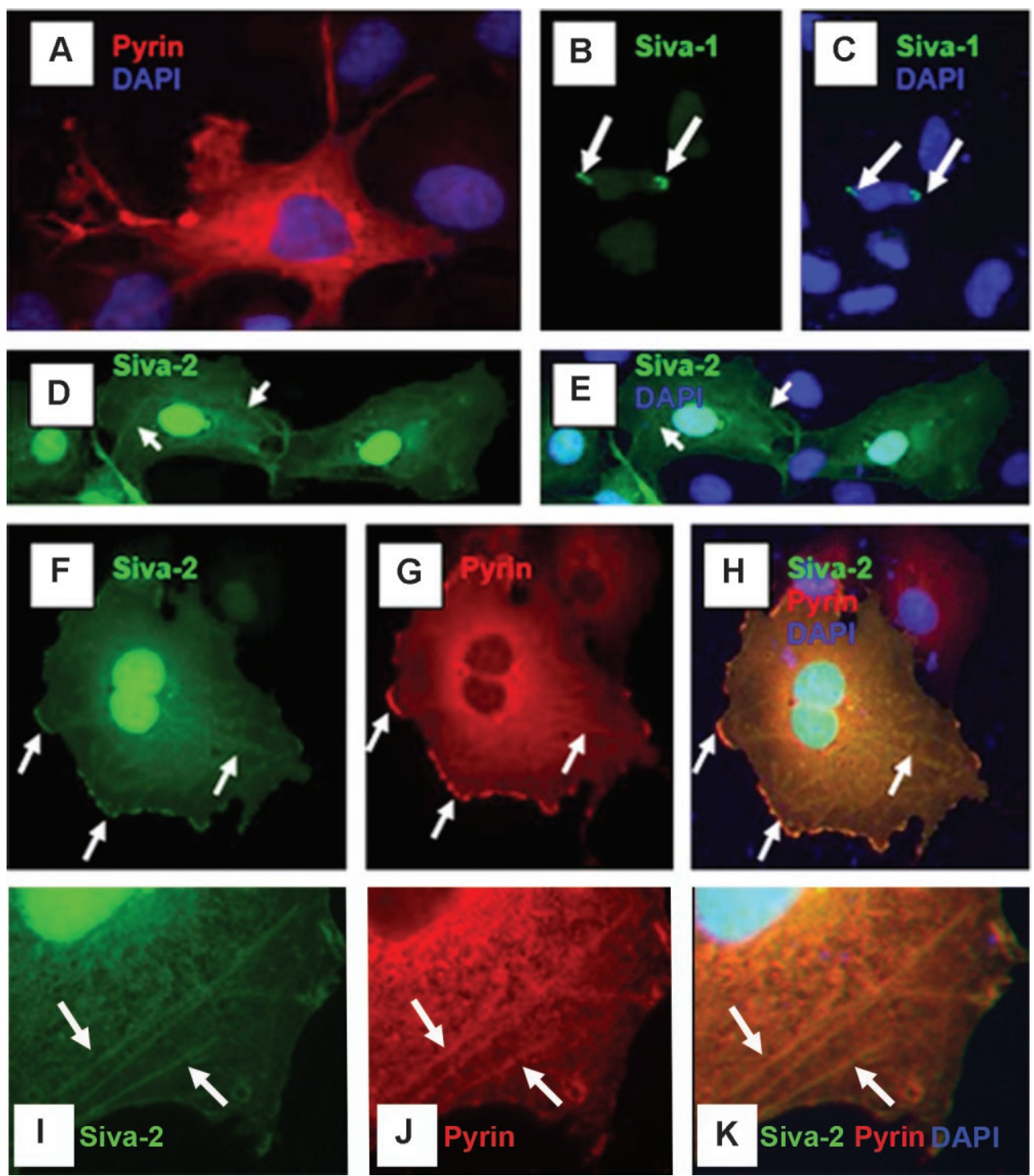

Fig. 4. Cellular localization of Siva and pyrin in co-transfected COS-7 cells. A: Myc-tagged pyrin is exclusively cytoplasmic. B,C: GFP-tagged Siva-I is nuclear; small nuclear aggregates are often seen (arrows). D,E: GFP-tagged Siva-2 is both nuclear and cytoplasmic. Cytoplasmic Siva-2 appears to be in stress fiber-like structures (arrows). F-H: Co-transfection of pyrin with Siva-2 results in a clear cytoplasmic co-localization of the proteins on the stress fiber-like structures in the cytoplasm and in lamellipodia-like structures at the membrane edge (arrows).

confirming pyrin-dependent attraction of Siva to ASC specks (data not shown). In addition, tests with another protein that does not interact with pyrin ( $\alpha$-actinin) showed that the attraction of Siva to pyrin-associated ASC was specific (data not shown). These findings not only provide further evidence for the binding of pyrin to both Siva-I and Siva-2, but also indicate that the triple association of pyrin-ASC and Siva is possible, allowing potential crosstalk between Siva and ASC-mediated signaling pathways.

\section{Interaction of Siva with pyrin constructs harboring FMF-associated mutations}

Many of the major FMF-associated mutations are clustered in the rfp domain of pyrin, and they have been recently proposed to affect the interaction between pyrin and Caspase-I (Chae et al., 2006). We therefore considered the possible effect of these mutations on the interaction between pyrin and Siva proteins. We examined the effect of the EI48Q mutation in pyrin exon 2, as well as the M694V mutation in exon 10 on Siva's ability to bind pyrin. Both GFP-tagged Siva-I and Siva-2 were precipitated with mutant forms of pyrin-myc at levels comparable to wild-type pyrin (Fig. 6), indicating no discernable effect of these mutations on binding in this assay.

\section{Effect of pyrin on Siva-induced apoptosis in response to oxidative stress}

Siva has been shown to be an apoptosis-associated protein in a variety of contexts (Prasad et al., 1997; Henke et al., 200I; Xue et al., 2002; Chu et al., 2004). It has further been reported that the two splice isoforms of Siva differentially transduce apoptosis; Siva-2 triggers apoptosis in Rat-I cells to a much lesser extent than Siva-I (Yoon et al., 1999). As we sought to identify a potential functional relevance for the interaction between pyrin and Siva, we examined whether this interaction 

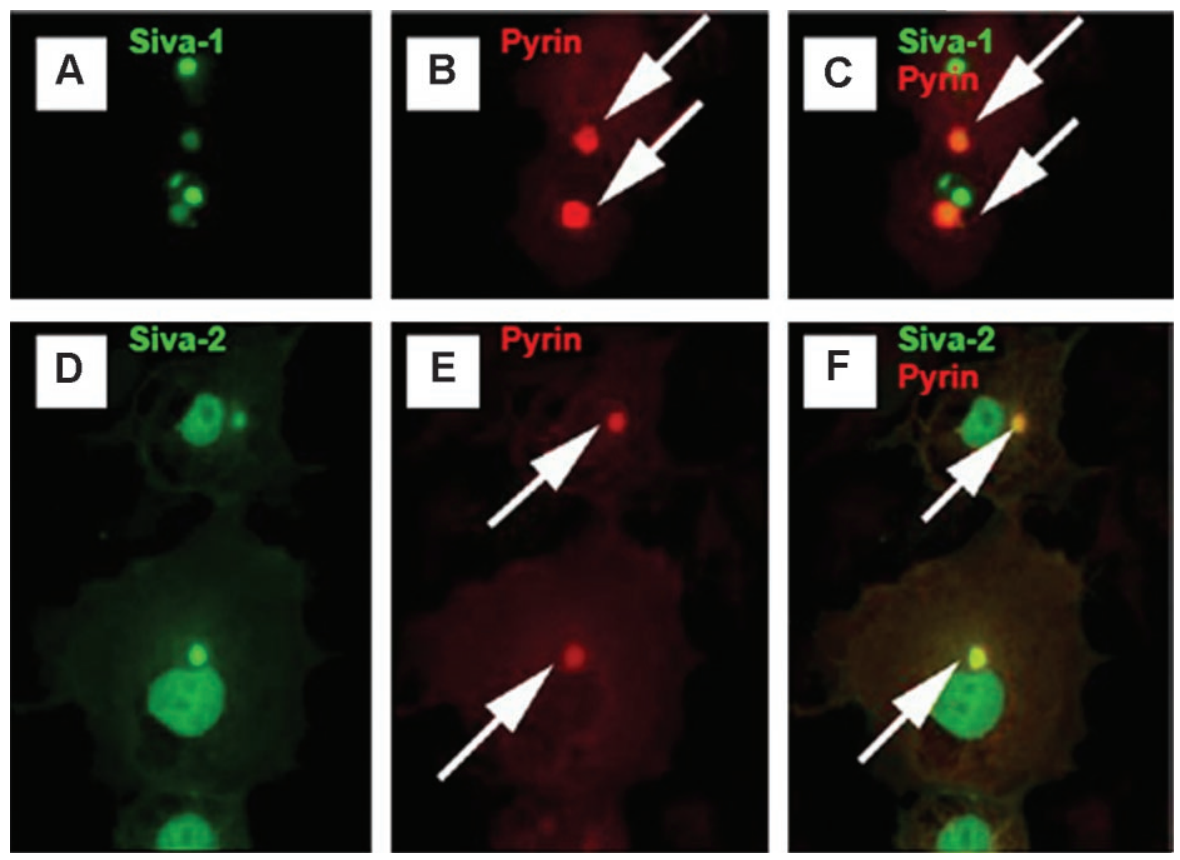

Fig. 5. Siva and pyrin co-localization in the ASC speck assay. A-C: COS-7 cells were co-transfected with GFP-tagged Siva-I (green, part A), myctagged pyrin (red, part B), and ASC (untagged). Part C is a merged picture of the red and green channels. Arrows point to ASC specks that contain pyrin in part B. Arrows in part C indicate that Siva-I is also located in these specks (yellow). D-F: Cells were co-transfected with GFP-tagged Siva-2 (green, part D), myc-tagged pyrin (red, partE), and ASC (untagged). Part F is a merged picture of the red and green channels. Arrows in part E point to ASC specks containing pyrin. Arrows in part F indicate that Siva-2 is also located in these specks (yellow).

alters Siva-mediated apoptosis. COS-7 cells were transfected with myc-tagged pyrin and GFP-tagged Siva, alone or in combination. The cells were then placed under oxidative stress by brief exposure to hydrogen peroxide, and the apoptotic response was assessed after $20 \mathrm{~h}$ as described in Materials and

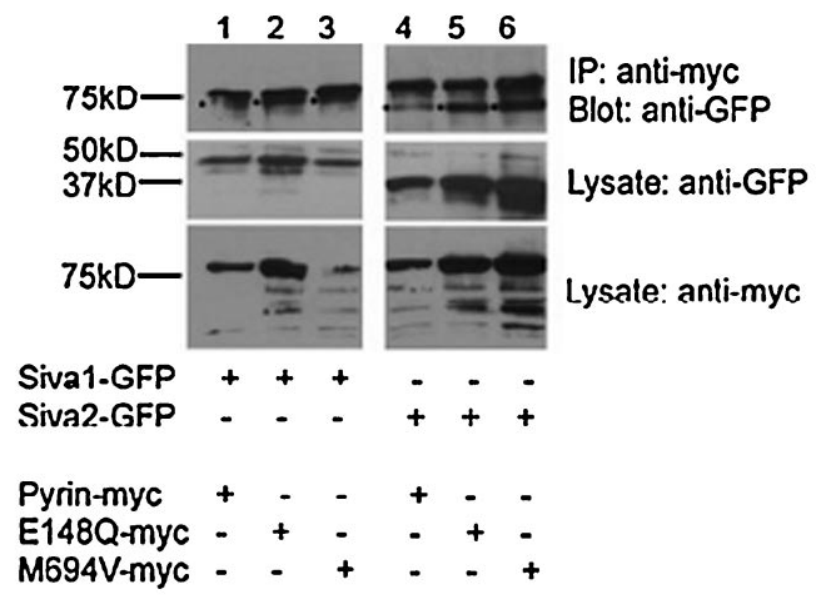

Fig. 6. Effect of pyrin mutations on the binding of Siva-I and Siva-2. The FMF-causing mutations EI 48Q and M694V were tested by immunoprecipitation for their ability to bind to Siva-I and Siva-2. No appreciable difference was discerned between the binding of wild-type and mutant forms of pyrin to Siva-I (lanes I-3) or Siva-2 (lanes 4-6). Dots to the left of the lanes indicate expected sizes of precipitated proteins.
Methods Section. The results from five independent replicates of this assay confirmed that wild-type pyrin, transfected alone, fails to induce a significant apoptotic response in control cells or in response to $\mathrm{H}_{2} \mathrm{O}_{2}$ (Fig. 7). Though Siva-I does not induce apoptosis in the absence of oxidative challenge, $\mathrm{H}_{2} \mathrm{O}_{2}$ exposure induces a clear apoptotic response, as described previously (Cao et al., 200I). However, co-transfection of Siva-I with pyrin significantly diminishes this Siva-induced apoptotic response.

In contrast to the results with Siva-I, Siva-2, when transfected alone, is unable to induce apoptosis in response to $\mathrm{H}_{2} \mathrm{O}_{2}$ in agreement with previous reports (Cao et al., 200 I). When Siva-2 and pyrin are co-transfected, a higher rate of apoptosis is observed than with either construct alone, but this effect, though reproducible, is not statistically significant.

We further explored these observations by using a disease-associated pyrin mutant (M694V) that contains a mutation in exon 10, the region shown to be important for the Siva-pyrin interaction. When M694V was transfected alone, it displayed a rate of apoptosis in response to $\mathrm{H}_{2} \mathrm{O}_{2}$ that was similar to that of wild-type pyrin (Fig. 7). Furthermore, when co-transfected with Siva-I, the M694V mutant pyrin, like wild-type pyrin, reduced the apoptotic response of Siva-I to $\mathrm{H}_{2} \mathrm{O}_{2}$ challenge, though in this case, the difference did not reach significance. As for wild-type pyrin, M694V pyrin did not modulate the Siva- 2 response to $\mathrm{H}_{2} \mathrm{O}_{2}$. Taken together, these observations suggest that pyrin may modulate apoptotic signaling transduced by Siva- I, but not by Siva- 2 .

\section{Discussion}

This article identifies and characterizes an interaction between pyrin and the proapoptotic protein, Siva. This brings the total number of known pyrin-interacting proteins to five: ASC, PSTPIPI, Caspase- I, I4-3-3, and Siva, further broadening the 


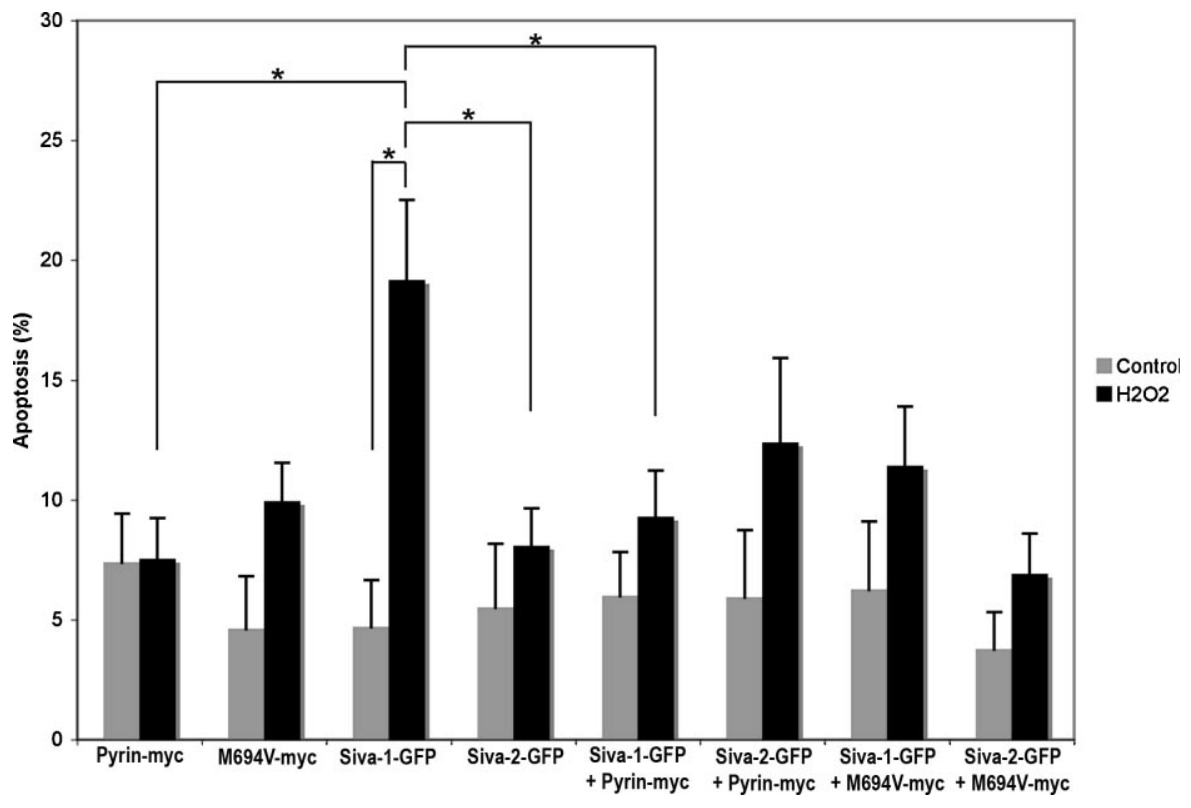

Fig. 7. Apoptotic response to oxidative stress. COS-7 cells were transfected with GFP-tagged versions of Siva and myc-tagged versions of pyrin, alone and in combination. The cells were then treated with hydrogen peroxide and examined for apoptosis. Siva-I caused a significant level apoptosis compared with control cells, while Siva-2 did not. When pyrin was co-transfected with Siva-I, the apoptotic activity of Siva was diminished. Asterisks indicate a significant change as determined by a $P$-value of less than 0.05 .

potential signaling role of pyrin in inflammation and apoptosis. The domain of pyrin that we find to be the most important for its interaction with Siva is the B30.2/SPRY/rfp domain that is encoded by exon 10 . This is the same region of the pyrin molecule that mediates its interaction with Caspase- $\mathrm{I}$. It is not clear at this time if the Caspase-I and Siva interactions are competitive or involve different regions of the B30.2/SPRY/rfp domain. Interestingly, ASC and PSTPIPI interaction domains of pyrin map to very different regions of the molecule ( $\mathrm{N}$-terminal PyD in the case of ASC and the B-box and coiled coil central region for PSTPIPI). We show here that pyrin can indeed bridge the co-localization of ASC and Siva, suggesting a mechanism by which ASC and Siva-mediated apoptotic signaling pathways could potentially intersect. Though it was not directly tested, the intersection of pyrin, Siva, and PSTPIPI also seems potentially possible.

We also present in this report a novel type of assay for the sensitive detection of cellular protein/protein interactions: the ASC speck recruitment assay. This assay relies on the efficient recruitment of pyrin's PyD to ASC specks. In this case, the fact that the PyD of pyrin seems dispensable for the pyrin/Siva interaction allowed us to use speck-associated pyrin as a cytosolic magnet to which Siva was attracted. Potentially, this system could be exploited for other protein/protein interaction tests, by: (a) linking the PyD of pyrin (approximately 90 amino acids) onto one of two potential interaction partners; (b) demonstrating effective recruitment of that PyD-tagged protein to ASC specks; and (c) testing whether triple transfection of ASC, the PyD tagged bait and its prey results in recruitment of the prey to specks. Since the speck represents a relatively large structure with a highly concentrated localization of ASC protein, the assay appears to be quite sensitive in detecting small amounts of potential prey proteins (in this case, Siva-I).

Our finding that pyrin's C-terminal rfp domain is important for its interaction with Siva is particularly interesting. The rfp domain is found in $>500$ different proteins of various functions; it has been proposed that this domain mediates protein/protein interactions or binds particular ligands (Woo et al., 2006; Papin et al., 2007). This domain has a particular importance for pyrin and FMF disease since the majority of FMF-associated mutations are localized to this region. When we examined the effect of two common mutations, we failed to find evidence that these mutations affect the binding interaction or the functional interaction in an oxidative stress assay. However, it is possible that the strength of the interactions is only mildly affected and cannot be measured with approaches or conditions used here. Since both pyrin and Siva interact dynamically with multiple other proteins, slight changes in binding affinity have the potential to alter the dynamics of cellular processes in which either protein participates.

In this regard, it is interesting that Siva also interacts with Arg kinase, a member of the c-Abl family of kinases. Arg phosphorylates both Siva-I and Siva-2 and this phosphorylation event is associated with the induction of apoptosis (Cao et al., 200I). Upon integrin ligation or cytokine exposure, both Abl and Arg become activated and localize to areas of p I03-CAS/ Crk assembly including focal adhesions and membrane ruffles (Kain and Klemke, 200I). Both Abl and Arg interact with the $\mathrm{SH} 3$ domain of Crk via their conserved polyproline region, and promote tyrosine phosphorylation of Crk-Y22I. Thus, Abl family kinases have an important biological role in the regulation of CAS/Crk coupling, an event that can be a switchpoint in the cell, determining whether the cell migrates or dies (Bouton et al., 200 I). Remarkably, both c-Abl and Crk bind PSTPIPI and are PEST phosphatase substrates (Cong et al., 2000). These observations suggest the possibility that a broader network of interactions between PSTPIPI and Siva pathways, potentially modulated by pyrin binding to both proteins, could affect both apoptosis and cell migration. It is interesting in this regard that pyrin and Siva-2 appear to co-localize at membrane ruffles. Since FMF is characterized by a massive migration of neutrophils into target inflammatory sites, an important next step will be to explore more fully the potential regulatory interactions between pyrin, Siva and PSTPIPI as well as the status of the Cas/Crk coupling switch in FMF patients between and during attacks. 
Another previous observation concerning Siva and pyrin may be of clinical importance given the physical and functional interactions between these two proteins documented here. It has been reported that the anti-cancer drug, cisplatin, can induce an FMF attack (Toubi et al., 2003). Interestingly, cisplatin induces a potent $\mathrm{p} 53$ response and Siva is one of only a few genes that are induced specifically during p53-mediated apoptosis (Jacobs et al., 2007). In fact, over-expression of Siva alone is sufficient to mimic p53-dependent cell death in the absence of p53 itself (Jacobs et al., 2007). Induction of p53 by Siva requires Caspase- 8 and involves the association of Siva with the plasma membrane (Jacobs et al., 2007), a compartment in which we also observe pyrin (Fig. 5). Further, the knockdown of Siva in cells treated with the DNA damaging toxin, camptothecin, attenuates the cell death response normally seen after treatment with this toxin. These findings together suggest the interesting hypothesis that induction of an FMF attack through cisplatin treatment might be Siva-dependent. The reduction in Siva-induced apoptosis in seen in the presence of pyrin (Fig. 7) could potentially permit a prolonged period of cellular inflammatory signaling through cytokine release that could trigger an attack. If this hypothesis is confirmed, the Siva-pyrin interaction might emerge as an important target for future therapeutic strategies.

\section{Acknowledgments}

The authors wish to thank Dr. Arturo Diaz for help with synoviocyte cultures and helpful discussions and Dr. Rengul Atalay for assistance. D.L.G. is grateful for support from NIH Al53262. Technical support from the Organogenesis Morphology Core is gratefully acknowledged.

\section{Literature Cited}

Bouton AH, Riggins RB, Bruce-Staskal PJ. 200I. Functions of the adapter protein Cas: Signa convergence and the determination of cellular responses. Oncogene 20:6448-6458.

Cao C, Ren X, Kharbanda S, Koleske A, Prasad KV, Kufe D. 200I. The ARG tyrosine kinase interacts with Siva-I in the apoptotic response to oxidative stress. J Biol Chem 276: I I 46511468.

Centola M, Aksentijevich I, Kastner DL. 1998. The hereditary periodic fever syndromes: Molecular analysis of a new family of inflammatory diseases. Hum Mol Genet 7:I58I-1588. Chae JJ, Wood G, Masters SL, Richard K, Park G, Smith BJ, Kastner DL. 2006. The B30.2 domain of pyrin, the familial Mediterranean fever protein, interacts directly with caspase- 1 to modulate IL-I beta production. Proc Natl Acad Sci USA 103:9982-9987.

Chu F, Borthakur A, Sun X, Barkinge J, Gudi R, Hawkins S, Prasad KV. 2004. The Siva-I putative amphipathic helical region $(S A H)$ is sufficient to bind to $B C L-X L$ and sensitize cells to UV radiation induced apoptosis. Apoptosis 9:83-95.

Chu F, Barkinge J, Hawkins S, Gudi R, Salgia R, Kanteti PV. 2005. Expression of Siva-I protein or its putative amphipathic helical region enhances cisplatin-induced apoptosis in breast cancer cells: Effect of elevated levels of BCL-2. Cancer Res 65:530I-5309.
Cong F, Spencer S, Cote JF, Wu Y, Tremblay ML, Lasky LA, Goff SP. 2000. Cytoskeletal protein PSTPIPI directs the PEST-type protein tyrosine phosphatase to the c-Abl kinase to mediate Abl dephosphorylation. Mol Cell 6:1413-1423.

Diaz A, Hu C, Kastner DL, Schaner P, Reginato AM, Richards N, Gumucio DL. 2004

Lipopolysaccharide-induced expression of multiple alternatively spliced MEFV transcripts in human synovial fibroblasts: A prominent splice isoform lacks the C-terminal domain that is highly mutated in familial Mediterranean fever. Arthritis Rheum 50:3679-3689.

Fernandes-Alnemri T, Wu J, Yu JW, Datta P, Miller B, Jankowski W, Rosenberg S, Zhang J, Alnemri ES. 2007. The pyroptosome: A supramolecular assembly of ASC dimers mediating inflammatory cell death via caspase-I activation. Cell Death Differ 14:1590-1604.

Fortin A, MacLaurin JG, Arbour N, Cregan SP, Kushwaha N, Callaghan SM, Park DS, Albert PR, Slack RS. 2004. The proapoptotic gene SIVA is a direct transcriptional target for the tumor suppressors p53 and E2 FI. J Biol Chem 279:28706-287/4.

Henke A, Nestler M, Strunze S, Saluz HP, Hortschansky P, Menzel B, Martin U, Zell R, Stelzne A, Munder T. 200I. The apoptotic capability of coxsackievirus B3 is influenced by the efficient interaction between the capsid protein VP2 and the proapoptotic host protein Siva. Virology 289:15-22.

Jacobs SB, Basak S, Murray JI, Pathak N, Attardi LD. 2007. Siva is an apoptosis-selective p53 target gene important for neuronal cell death. Cell Death Differ 14:1374-1385.

Jeru I, Papin S, L'Hoste S, Duquesnoy P, Cazeneuve C, Camonis J, Amselem S. 2005. Interaction of pyrin with 14.3 .3 in an isoform-specific and phosphorylation-dependent manner regulates its translocation to the nucleus. Arthritis Rheum 52:1848-1857.

Kain KH, Klemke RL. 200I. Inhibition of cell migration by Abl family tyrosine kinases through uncoupling of Crk-CAS complexes. J Biol Chem 276:16185-16192.

Livhen A, Lengevitz P, Zewer D. 1997. Criteria for the diagnosis of FMF. Arthritis Rheum 40:1879-1885.

Martinon F, Burns K, Tschopp J. 2002. The inflammasome: A molecular platform triggering activation of inflammatory caspases and processing of prolL-beta. Mol Cell 10:417-426.

Masumoto J, Taniguchi S, Ayukawa K, Sarvotham H, Kishino T, Niikawa N, Hidaka E, Katsuyama T, Higuchi T, Sagara J. 1999. ASC, a novel 22-kDa protein, aggregates durin apoptosis of human promyelocytic leukemia HL-60 cells. J Biol Chem 274:33835-33838.

Nestler M, Martin U, Hortschansky P, Saluz HP, Henke A, Munder T. 2006. The zinc containing pro-apoptotic protein siva interacts with the peroxisomal membrane protein pmp22. Mol Cell Biochem 287:147-I55.

Papin S, Cuenin S, Agostini L, Martinon F, Werner S, Beer HD, Grutter C, Grutter M, Tschopp J. 2007. The SPRY domain of Pyrin, mutated in familial Mediterranean fever patients, interacts with inflammasome components and inhibits prolL-l beta processing. Cell Death Differ 14:1457-1466.

Prasad KV, Ao Z, Yoon Y, Wu MX, Rizk M, Jacquot S, Schlossman SF. 1997. CD27, a member of the tumor necrosis factor receptor family, induces apoptosis and binds to Siva, a proapoptotic protein. Proc Natl Acad Sci USA 94:6346-635I.

Py B, Slomianny C, Auberger P, Petit PX, Benichou S. 2004. Siva-I and an alternative splice form lacking the death domain, Siva-2, similarly induce apoptosis in T lymphocytes via a caspase-dependent mitochondrial pathway. J Immunol 172(7):4008-4017.

Richards N, Schaner P, Diaz A, Stuckey J, Shelden E, Wadhwa A, Gumucio DL. 2001. Interaction between pyrin and the apoptotic speck protein (ASC) modulates ASC-induced apoptosis. J Biol Chem 276:39320-39329.

Schaner PE, Gumucio DL. 2005. Familial Mediterranean fever in the post-genomic era: How an ancient disease is providing new insights into inflammatory pathways. Curr Drug Targets Inflamm Allergy 4:67-76.

Shoham NG, Centola M, Mansfield E, Hull KM, Wood G, Wise CA, Kastner DL. 2003. Pyrin binds the PSTPIPI/CD2BPI protein, defining familial Mediterranean fever and PAPA syndrome as disorders in the same pathway. Proc Natl Acad Sci USA 100:1350 I-13506.

Spinicelli S, Nocentini G, Ronchetti S, Krausz LT, Bianchini R, Riccardi C. 2002. GITR interacts with the pro-apoptotic protein Siva and induces apoptosis. Cell Death Differ 9:1382-1384. Toubi E, Gershoni-Baruch R, Kuten A. 2003. Cisplatin treatment triggers familial Mediterranean fever attacks. Tumori 89:80-81.

Wise CA, Gillum JD, Seidman CE, Lindor NM, Veile R, Bashiardes S, Lovett M. 2002 Wise CA, Gillum JD, Seidman CE, Lindor NM, Veile R, Bashiardes S, Lovett M. 2002
Mutations in CD2BPI disrupt binding to PTP PEST and are responsible for PAPA syndrome, an autoinflammatory disorder. Hum Mol Genet II:96I-969.

Woo JS, Imm JH, Min CK, Kim KJ, Cha SS, Oh BH. 2006. Structural and functional insights into the B30.2/SPRY domain. EMBO J 25:1353-1363.

Xue L, Chu F, Cheng Y, Sun X, Borthakur A, Ramarao M, Pandey P, Wu M, Schlossman SF, Prasad KV. 2002. Siva-I binds to and inhibits BCL-X(L)-mediated protection against UV radiation-induced apoptosis. Proc Natl Acad Sci USA 99:6925-6930.

Yoon Y, Ao Z, Cheng Y, Schlossman SF, Prasad KV. 1999. Murine Siva-I and Siva-2, alternate splice forms of the mouse Siva gene, both bind to CD27 but differentially transduce apoptosis. Oncogene 18:7174-7179. 